\title{
Response to Margit Warburg
}

RUTH ILLMAN

The Donner Institute

\author{
MERCÉDESZ CZIMBALMOS \\ Åbo Akademi University
}

We have received and read Professor Margit Warburg's response to our article with sincere reflection and self-assessment. We regret the shortcomings of the information retrieval process and acknowledge that Professor Warburg's contributions to the conceptualization of the field are indeed valuable and noteworthy. We also acknowledge that she mentioned this to us in a comment on our presentation at the EASR conference in Tartu in 2019, and that we later failed to follow up on it.

It is perhaps always a sign of hubris to employ the words 'novel' and 'new' in a research article, and in retrospect it would have been wise to underscore more clearly the following important caveat (p. 171 and 173): 'The analytical model contributes to the study of vernacular religion by bringing together major themes recognized as relevant in previous research in a structured theoretical and methodological framework and by offering an analytical tool for detecting these themes in ethnographic materials'. This phrase is included in the abstract, which is one of the most important identifiers in commonly used research repositories and information retrieval systems, along with the title, authors, and keywords. This means that our caution should be readily noticed by anyone happening on our article in their information search. However, we acknowledge in response to Warburg's reading that this statement received too little prominence in the argument, skewing it towards the novelty aspect, which should not have been the focus.

Our main objective in this article was to contribute to the ethnographic research within the study of contemporary religions. Thus, the fields of sociolinguistics, leadership training, and educational or even quantitative research within the sociology of religion, for example, were not the main 
scope of our reference work. However, we do acknowledge that an excellent study should of course have described and credited all the related fields in which similar terminology had been employed. However, within the limits of a single article this was an insurmountable task.

This underlines the validity of Warburg's observation that in contemporary digital information retrieval processes it is challenging to discover information sources that do not use the keywords at which you target your search as relevant indexing identifiers, that is, in the publication's title, abstract, and keywords. This is especially true of monographs and edited volumes such as those mentioned by Warburg, which clearly offer relevant perspectives on our research theme but have not been indexed in a way that makes them retrievable in a search based on the keywords we used. The concepts of knowing, being, and doing do not appear in the title of Warburg's monograph dealing with the matter, Citizens of the World: A History and Sociology of the Baha'is from a Globalisation Perspective (Leiden: Brill, 2006), nor in any of its subtitles, abstract, or index terms; they are embedded in Chapter 8, where they leave only faint or no traces in the metadata.

We therefore share Warburg's concern about the increasing dominance of digital publications in our scholarly milieu and the likewise increasing impermeability of the information landscape in which researchers of religion and neighbouring disciplines today are navigating. This flood of information makes it increasingly important for researchers to delimit their objectives and the claims made in their research. The concepts of knowing, being, and doing (which in our simple model can be used in any order or combination) are indeed generic and have thus been used in a great many studies of religion, as exemplified by Warburg. This makes the task of delimiting the discussion in any given article on the theme even more important, as well as clearly indicating which scholarly discussions one regards oneself as participating in and advancing. This became one of our most demanding challenges during the editorial process.

We were rightly criticized by our two anonymous peer reviewers for the broad span of our sources, and strongly advised to streamline and solidify our argument by focusing our references on a more particular area of research. We therefore focused only on scholars who employed the term 'vernacular religion' in their research and regarded their research as contributions to this particular ethnographic tradition (in particular, as an alternative to the more widely used notion of 'lived religion'). Furthermore, we focused on researchers dealing with contemporary Judaism and Jewishness, apart from the section in which we gave examples of various cultural and religious contexts in which the term 'vernacular religion' has been attempted. It was unfortunate that Warburg's excellent study evaded our attention because of its focus on 
quantitative analysis, the study of the Baha'is, and its disciplinary rootedness in the epistemological traditions of the sociology of religion. In hindsight, the same can be said of the other examples listed by Warburg: they are indeed examples of prominent research using the generic concepts, but not from the perspective of vernacular religion, ethnographic research, or Judaism, which are the sources of our argument.

Finally, in response to the suspicion that all future researchers will feel required to reference our article we would like to adopt a more modest perspective. As a trained librarian (Illman) and an archivist with vast experience of processing big data (Czimbalmos), we would in the first case advise any researcher not to use Google Scholar as their main tool for information searches, even if it has a strong impact on the visibility of scholarship, as Warburg stresses. We would attach less significance to the fact that algorithmic research tools currently pick up our article in Warburg's search: these results are constantly changing and depend on algorithms that are arbitrary, biased, and substantially shaped by previous information behaviour, location, commercial interests, and chance. Against this background researchers can easily omit our article from their future reference lists if our focus is of no immediate relevance to them. Likewise, we hope that researchers seeking to contribute to other epistemological traditions and conceptual developments within the broad span of the study of religions find their way to the very important studies indicated by Warburg.

We would again like to thank Professor Warburg for her substantial and valid remarks concerning our analysis, and acknowledge her long experience and strong record of excellent scholarship and publishing in the study of religions, as well as the carelessness of our review of previous research in the field. While we have not referenced her work duly in the current contribution, we have amended this shortcoming in our subsequent work in forthcoming publications. Most importantly, we welcome the broad discussion, so eloquently aired by Margit Warburg, of the growing challenges each researcher of religion, or any other discipline, faces today in seeking to conduct solid and informed reviews of previous research in an increasingly unmanageable information environment.

$* * *$

RUTH ILLMAN is associate professor of the study of religions (Åbo Akademi University) and history of religions (Uppsala University), and the director of the Donner Institute for Research in Religion and Culture, Åbo, Finland. Email: ruth. illman@abo.fi

MERCÉDESZ CZIMBALMOS PhD is a project researcher in the 'Manifold more' project at the Finnish Institute for Health and Welfare, Helsinki, Finland. Email: mercedesz.czimbalmos@abo.fi 
